\title{
LETTER \\ Performance Evaluation of CDMA Using Chaotic Spreading Sequence with Constant Power in Indoor Power Line Fading Channels
}

\author{
Ryo TAKAHASHI ${ }^{\dagger a)}$ and Ken UMENO ${ }^{\dagger \dagger}$, Members
}

SUMMARY In this study, a performance of a synchronous code division multiple access (CDMA) using the chaotic spreading sequences with constant power is estimated in indoor power line fading channels. It is found that, in the fading channels, as the number of simultaneous users increases, the chaotic spreading sequences realize better performance than the Walsh-Hadamard sequences in the synchronous CDMA.

key words: chaos, CDMA, fading, power line channel

\section{Introduction}

Code division multiple access (CDMA) is a spread spectrum multiple access technique which is widely used in communication system. This multiple access is realized by assigning spreading sequences to each simultaneous signal. The properties of the spreading sequences affect the performance of CDMA communication system significantly. Therefore, a design of spreading sequences is a mature field [1]-[11]. Recently, a CDMA, to which a real-valued complex chaotic time series with constant power is applied as the spreading sequence, has been proposed and evaluated to realize a highcapacity and more secure communication link [10], [11]. It was found that this technique utilizing chaos increases a capacity of the number of simultaneous connections. These evaluations are assumed to be under non-dispersive channels. In practice, most of channels for communications have fading characteristics not only in wireless communication but also in wired one. Therefore, the performance evaluation in the fading channels is also necessary toward practical usage.

On the other hand, power line communication (PLC) system attracts much attention as one of the key technologies of smart grids [12]. The PLC is a wired communication system which transmits the signal over power lines. In the smart grid environment, real-time information of energy consumed by appliances is acquired using highly developed information and communications technologies. These appliances are inevitably connected to power lines. Therefore, the PLC has attracted much interest for the acquisition of

\footnotetext{
Manuscript received November 20, 2013.

Manuscript revised March 11, 2014.

$\dagger$ The author is with the Graduate School of Engineering, Kyoto University, Kyoto-shi, 615-8510 Japan.

${ }^{\dagger}$ The author is with the Graduate School of Informatics, Kyoto University, Kyoto-shi, 606-8501 Japan.

a) E-mail: takahashi.ryo.2n@kyoto-u.ac.jp

DOI: 10.1587/transfun.E97.A.1619
}

information and the control of appliances [12]-[14]. The power line channel is one of the representative fading channels because an impedance mismatching causes signal reflections.

In this study, the performance of synchronous CDMA using complex chaotic spreading sequences with constant power is investigated in indoor power line fading channels, as one of the fading channels. Its performance was compared with that of the one using the Walsh-Hadamard (WH) sequences, which have complete orthogonality in an ideal synchronous system, and which are used for practical CDMA. Here, the performance of the chaotic series as the spreading sequence for CDMA is focused on. Error control codes are not applied. In the power line fading channels, the bit error rates (BERs) are estimated by performing numerical simulations. This paper is organized as follows. In Sect. 2, the fundamental properties of the complex chaotic spreading sequences are explained. In Sect. 3, the power line fading channels used here are explained in detail. In Sect. 4, the simulated system and parameter settings are explained. The BERs obtained by the numerical simulation are estimated and discussed. Finally, the conclusion is given in Sect. 5.

\section{Complex Chaotic Spreading Sequences with Con- stant Power}

The complex chaotic spreading sequence $\left\{Z_{i}\right\}$ (i= $0,1, \cdots N)$ used here is composed of In-phase and Quadrature-phase real-valued sequences. The sequences can be obtained by using the Chebyshev polynomials [10]. The quantity $Z_{i}$ can be represented as $Z_{i}=X_{i}+j Y_{i}$, where $j$ indicates an imaginary unit. A part of the sequences, e.g., In-phase $X_{i}$, is generated by the following relation:

$$
X_{q, i+1}=T_{q}\left(X_{q, i}\right), \quad T_{q}(\cos \theta)=\cos (q \theta),
$$

where $T_{q}(x)$ is the $q$-th order Chebyshev polynomial and $q \geq 2, q \in N$. It is known that the Chebyshev map $T_{q}(x)$ is ergodic, and it has an ergodic invariant measure given by $\rho(x) d x=d x /\left(\pi \sqrt{1-x^{2}}\right)$. It satisfies the relation given by $\int_{-1}^{1} T_{i}(x) T_{j}(x) \rho(x) d x=\delta_{i, j}\left(1+\delta_{i, 0}\right) / 2$, where $\delta_{i, j}$ is the Kronecker delta function. This relation indicates a statistical orthogonality between the sequences. It is also known that a Lyapunov exponent of the sequence generated by $T_{q}(x)$ is 
given by $\log q$. Conversely, another part of the sequence $Y_{j}$ is generated by

$$
Y_{q, i+1}=T_{q}^{\prime}\left(X_{q, i}, Y_{q, i}\right), \quad T_{q}^{\prime}(\cos \theta, \sin \theta)=\sin (q \theta) .
$$

The invariant measure of $\left\{Y_{i}\right\}$ is the same as that of $\left\{X_{i}\right\}$. It is easy to show that the sequences can realize a constant power as follows: $\left|Z_{q, i}\right|^{2}=X_{q, i}^{2}+Y_{q, i}^{2}=\cos ^{2}(q \theta)+\sin ^{2}(q \theta)=1$. Finally, the complex chaotic sequence with constant power $\left\{Z_{i}\right\}$ can be generated by the following relation:

$$
Z_{q, i+1}=F\left(Z_{q, i}\right), \quad F\left(e^{j \theta}\right)=e^{j \theta \theta} .
$$

The invariant measure for the transformation $z \rightarrow F(z)=z^{q}$ is represented by the following uniform measure: $\rho(z) d z=$ $d \theta /(2 \pi)$. The sequences applied to each channel or user can be obtained by changing these initial values $X_{0}$ and $Y_{0}$ or the order $q$. The performance of complex chip-synchronous CDMA using the above chaotic spreading sequences has been investigated in detail for the system with an additive white Gaussian noise (AWGN) channel [11]. In this study, we apply the sequences $X_{q, j}$ and $Y_{q, j}$ to I (In-phase) and Q (Quadrature-phase) channels, respectively.

\section{Power Line Fading Channel}

Here, we use a power line channel model proposed by Tsuzuki et al. [15]. The power line is a VVF (Vinyl insulation, Vinyl sheath, Flat) electrical cable with two wires of $\phi 1.6 \mathrm{~mm}$, which is widely used in Japan for indoor power lines. One of the configurations of the channels is shown in Fig. 1. The signal transmitter and receiver are set at points $A$ and $\mathrm{D}$, respectively. There are two branches at points $\mathrm{B}$ and $\mathrm{C}$, while the appliances can be connected at points $\mathrm{E}$ and $\mathrm{F}$. Impedance mismatching causes signal reflection, which results in a frequency selective fading response in the power line channel. A practical model of the transfer function is developed for VVF cable by adopting the measured parameters.

In this study, we consider two configurations on the basis of the model proposed in [15]. One is the branched configuration shown in Fig. 1. The other is a branch-less configuration, which is constructed by one stretched VVF cable between the points $\mathrm{A}$ and $\mathrm{D}$. The transfer function for this branch-less configuration can be obtained by adopting the parameter used for the branched configuration. We assume that the impedances are matched along the cable, transmitter, and receiver. In the branched configuration, openended branches with no appliances are assumed. Figure 2 shows the transfer functions as functions of the frequency for the branched and branch-less configurations mentioned above. From the figure, it is observed that the gain for the branch-less channel decreases monotonically, and the one for branched channel decreases non-monotonically and drops sharply around a particular frequency. At the same time, for the branched configuration, nonlinear phase characteristics are shown. From these results, it is confirmed that the branched power line channel has a frequency-selective

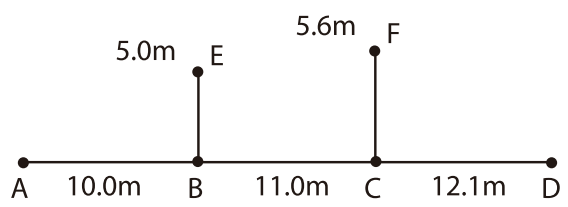

Fig. 1 Transmission line composed of VVF cables.

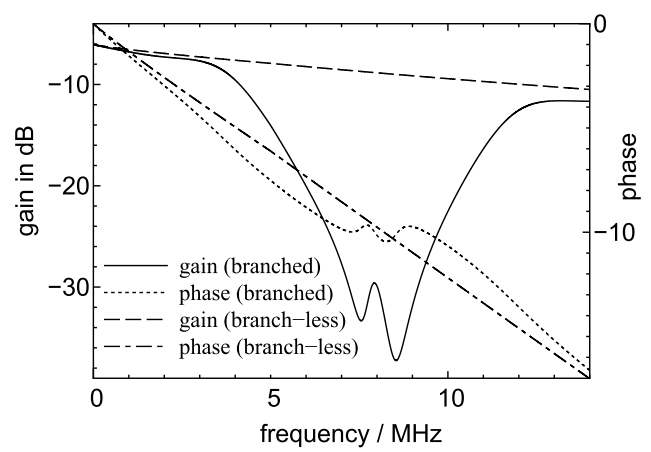

Fig. 2 Transfer functions for the power line channels investigated in this study. Solid and dash lines represent gains for branched and branch-less power lines, respectively. Dot and dot dash lines represent phase responses for these lines, respectively.

fading response. In addition, even the branch-less channel has a low-pass response. Here, since the performance of the chaotic spreading sequences in the fading channels is focused on, we do not consider an impulsive noise which is one of the characteristics of the power line communication system [13].

\section{Bit Error Rate Obtained by Numerical Simulation}

In this study, the performance of a synchronous CDMA using the chaotic spreading sequences with constant power is evaluated in the power line fading channels explained in the previous section. The performance is compared with that of the one using the WH sequences, which is well known to possess complete orthogonality in an ideal synchronous system. Figure 3 shows a schematic diagram of the investigated communication system, which focuses on one user. Here, $b$ and $C$ indicate the transmitted bit \pm 1 and spreading sequences, respectively. In the multi-user case, all users' signals are combined in one transmitted signal, and the signal is inputted to the channel $H(f)$. Each user uses both I and Q channels, and raised cosine filters are set. The bandwidth is set to $(1+\alpha) / T_{c}$, where the chip rate $1 / T_{c}$ is 5 Mcps (chip per second) and the roll-off factor $\alpha$ is 0.5. The channel $H(f)$ is the power line channel shown in Fig. 2. The quantity $\varphi$ is a phase delay caused by this channel. The white Gaussian noise is added to the signal after passing through $H(f)$. The carrier frequency $\omega_{\mathrm{c}}$ was set to $2 \mathrm{MHz}$ since frequencies between 2 and $30 \mathrm{MHz}$ are used for high-speed power line communication. The number of users was set to two and the spreading factor $N$ was set to 128 . In the case where $N=128$, the number of obtainable WH sequences is 128 . For every set of simulations, four different sequences were 


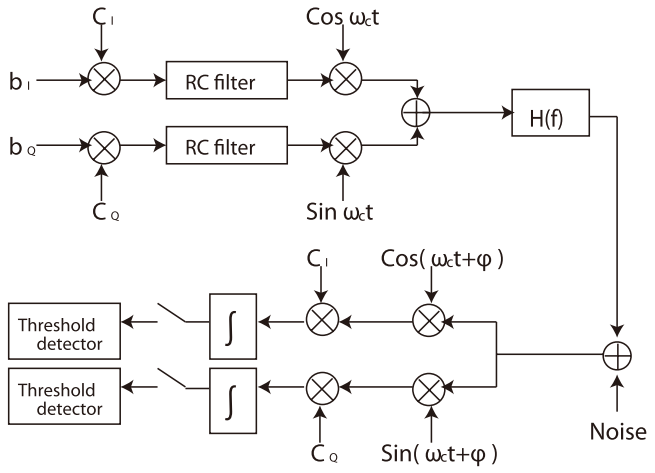

Fig. 3 Schematic diagram of the investigated communication system.

randomly extracted from these 128 sequences. In the case where the chaotic spreading sequences are used, the initial values $X_{0}$ and $Y_{0}$ were randomly set for every set. These four randomly-selected sequences were applied to each of the I/Q channels for two users. For one set of simulations, 40 bits for each channel were transmitted, i.e., a total of $40 \times 4$ bits (two users and I/Q channels) were transmitted. The bits were randomly set to \pm 1 . We obtained a distribution of sampled correlator output data before the input to a threshold detector. The BERs for each set were estimated numerically by applying the standard Gaussian approximation to the distribution. Finally, 40 sets of simulations were executed.

Figure 4 shows the BERs as functions of bit energy at a transmitter per noise power $E_{b} / N_{0}$. The BERs for the branched and branch-less power line channels are shown in Figs. 4(a) and (b), respectively. Each point indicates the mean value for 40 sets. The error bars indicate the standard deviations, which are obtained after applying a common logarithm to each of these 40 BERs. From this figure, it is found that the mean BERs of the system with the chaotic spreading sequences are better than the ones with the WH sequences. In addition, with regards to the standard deviation, there are large differences between the chaotic and WH sequence. While there is a large deviation for the WH sequences, the deviation for the chaotic sequences was found to be much narrower. This result implies that the performance for the chaotic spreading sequences does not depend on the choice of these sequences, such as the choice of the initial values for the chaotic sequences $X_{0}$ and $Y_{0}$. On the contrary, the choice from the limited number of WH sequences (which is 128 sequences in this study), significantly affects the BER. This is because the chaotic spreading sequences possess a much smaller difference each other in the power spectral distribution when compared with the WH sequences. Figures 5(a) and (b) show examples of the power spectral distributions of the transmitted signal just before input to the power line channel $H(f)$ with the WH sequences and that with the chaotic spreading sequences, respectively. The transmitted signal bundles four-channel signals to which the different spreading sequences were applied. It is found that there are dense and sparse areas in the
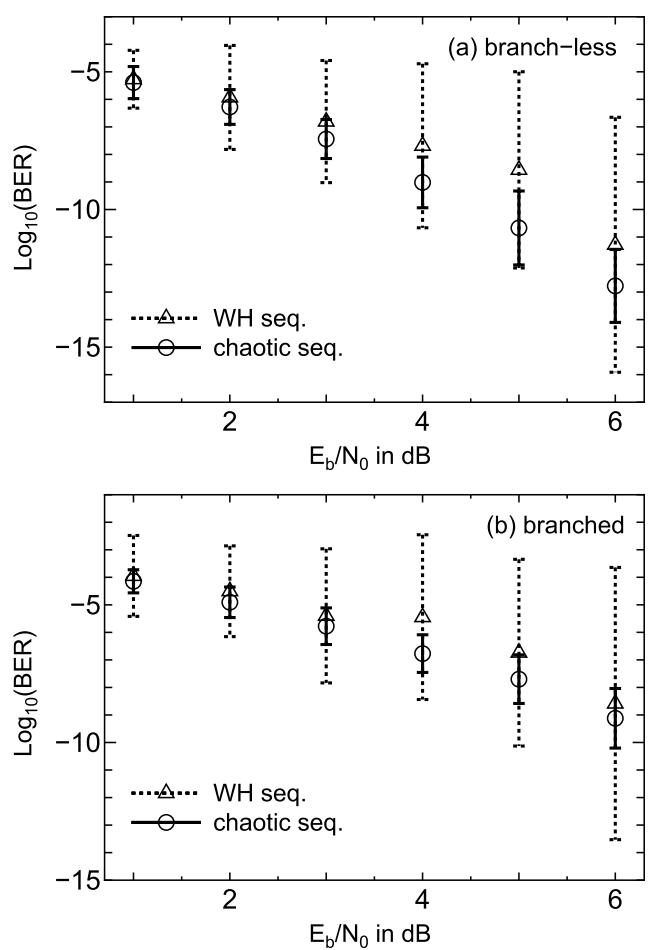

Fig. 4 BERs in synchronous CDMA as functions of bit energy at transmitter over noise power $E_{\mathrm{b}} / N_{0}$ in (a) branch-less and (b) branched power line fading channels. The complex chaotic spreading sequences with constant power or Walsh-Hadamard spreading sequences are used.
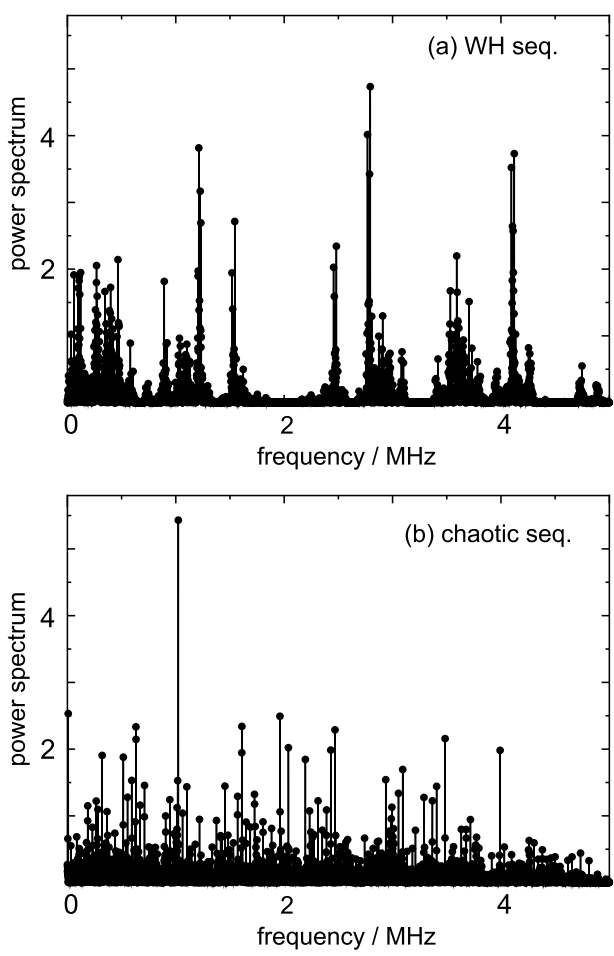

Fig.5 Examples of the power spectral distributions of the transmitted signal just before input to the power line channel for (a) Walsh-Hadamard sequences and (b) chaotic sequences. 
spectrum of the transmitted signal with the WH sequences. We confirmed that the locations of the peaks are different for each WH sequence. Conversely, the distribution for the chaotic sequences was almost uniform, as opposed to the one for the WH sequences. The shapes of the spectrum for other chaotic sequences were similar to those in this example. In the channels which have non-uniform frequency characteristics such as the power line channels, the shape of the spectrum of the transmitted signal affects the performance of the system largely. Finally, on the basis of these results, it was found that the system with the chaotic spreading sequences has a BER close to the mean value without the selection of these sequences. Conversely, for the WH sequences, it was necessary to select the sequences that are appropriate to the channel response. The WH sequences appropriate to the channel realize a significantly low BER; however, it would be more difficult to select the matched sequences as the number of users increase.

\section{Conclusion and Discussion}

In this study, the performance of synchronous CDMA using the complex chaotic spreading sequences with constant power was estimated in power line fading channels. The performance of the chaotic spreading sequences for CDMA was compared with that of the WH sequences. It was found that the chaotic spreading sequences for CDMA realize lower mean BERs and much narrower standard deviation of BERs than the WH sequences in both the branch-less and branched power line fading channels. This result implies that, as the number of users increases, the chaotic spreading sequences realize a better performance when compared with the WH sequences in the fading channels.

In order to evaluate the performance as the PLC system, it would be necessary to take into account the asynchronous impulsive noise which is one of the features of the power line channels [13]. In addition, as a communication system, several techniques such as error control coding should be applied. Here, we focus on the performance of the chaotic spreading sequence for CDMA in the fading channels. The reason of the performance improvements in the fading channels by chaotic spreading sequences is the shape of power spectrum of spread signals. The spectrum of the chaotic spreading sequences are almost uniform like white noise, as opposed to the one for the WH sequences. Therefore, we conclude that the chaotic spreading sequence for CDMA can realize high-capacity communication in fading channels. Gold sequence is also well known as the one used in the practical asynchronous CDMA. The power spectrum of Gold sequence is similar to the white noise as well as the one of the chaotic sequence. However, the chaotic sequence can be expected to realize the lower BER in the asynchronous CDMA than the Gold sequence. This is because the chaotic sequence realizes better Signal-to-Interference Ratio than the Gold sequence in the chip-synchronous system which is close to the asynchronous system [8]. The chaotic spreading sequence with constant power for CDMA is highly expected to realize a more secure and higher capacity communication.

\section{Acknowledgment}

The author (R.T.) was partially supported by the JSPS, Grant-in-Aid for Young Scientists (B), 24700224, 2013, and the NICT. The author (K.U.) was partially supported by the competitive fund No. 120829003 of the Ministry of the Environment, Japan. We appreciate fruitful discussion with Prof. Takashi Hikihara and the members in Advanced Electrical Systems Theory Laboratory in Kyoto University.

\section{References}

[1] G.N. Karystinos and D.A. Pados, "New bounds on the total squared correlation and optimum design of DS-CDMA binary signature sets," IEEE Trans. Commun., vol 51, pp.48-51, Jan. 2003.

[2] D.Y. Peng and P.Z. Fan, "Generalized Sarwate bounds on the aperiodic correlation of sequences over complex roots of unity," Proc. IEE Commun., vol.151, pp.375-382, Aug. 2004.

[3] T. Kohda and A. Tsuneda, "Pseudonoise sequences by chaotic nonlinear maps and their correlation properties," IEICE Trans. Commun., vol.E76-B, no.8, pp.855-862, Aug. 1993.

[4] T. Kohda and A. Tsuneda, "Statistics of chaotic binary sequences," IEEE Trans. Inf. Theory, vol.43, pp.104-112, Jan. 1997.

[5] G. Mazzini, G. Setti, and R. Rovatti, "Chaotic complex spreading sequences for asynchronous DS-CDMA.I. System modeling and results," IEEE Trans. Circuits Syst. I, vol.44, pp.937-947, Oct. 1997.

[6] R. Rovatti, G. Setti, and G. Mazzini, "Chaotic complex spreading sequences for asynchronous DS-CDMA. II. Some theoretical performance bounds," IEEE Trans. Circuits Syst. I, vol.45, pp.496-506, April 1998.

[7] K. Umeno and K. Kitayama, "Spreading sequences using periodic orbits of chaos for CDMA," Electron. Lett., vol.35, no.7, pp.545$546,1999$.

[8] K. Umeno and K. Kitayama, "Improvement of SNR with chaotic spreading sequences for CDMA," Proc. 1999 IEEE Information Theory Workshop, p.106, 1999.

[9] C.C. Chen, K. Yao, K. Umeno, and E. Biglieri, "Design of spreadspectrum sequences using chaotic dynamical systems and ergodic theory," IEEE Trans. Circuits Syst. I, vol.48, pp.1110-1114, Sept. 2001

[10] K. Umeno, "On the complex chaotic spreading sequence with constant power," IEICE Technical Report, NLP2003-188, 2003.

[11] R. Takahashi and K. Umeno, "Performance analysis of complex CDMA using complex chaotic spreading sequence with constant power," IEICE Trans. Fundamentals, vol.E92-A, no.12, pp.33943397, Dec. 2009

[12] S. Galli, A. Scaglione, and Z. Wang, "For the grid and through the grid: The role of power line communications in the smart grid," Proc. IEEE, vol.99, no.6, pp.998-1027, June 2011.

[13] M. Katayama, T. Yamazato, and H. Okada, "A mathematical model of noise in narrowband power line communication systems," IEEE J. Sel. Areas Commun., vol.24, no.7, pp.1267-1276, July 2006.

[14] T. Takuno, Y. Kitamori, R. Takahashi, and T. Hikihara, "AC power routing system in home based on demand and supply utilizing distributed power sources,” Energies, vol.4, no.5, pp.717-726, 2011.

[15] S. Tsuzuki, T. Takamatsu, H. Nishio, and Y. Yamada, "An estimation method of the transfer function of indoor power-line channels for Japanese houses," Proc. IEEE International Symposium on PowerLine Communications and Its Applications (ISPLC2002), pp.55-59, 2002 